LETTER

Elastic collision and breather formation of spatiotemporal vortex light bullets in a cubicquintic nonlinear medium

To cite this article: S K Adhikari 2017 Laser Phys. Lett. 14065402

View the article online for updates and enhancements.

\section{Related content}

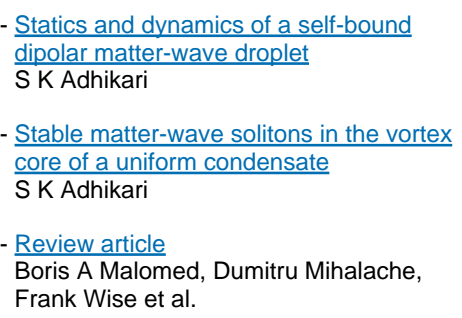

Statics and dynamics of a self-bound dipolar matter-wave droplet S K Adhikari

- Stable matter-wave solitons in the vortex core of a uniform condensate S K Adhikari

- Review article

Boris A Malomed, Dumitru Mihalache, Frank Wise et al.

\section{Recent citations}

- Lattice and quintic nonlinearity induced
stripe phase in Bose-Einstein condensate
under non-inertial and inertial motion
Priyam Das

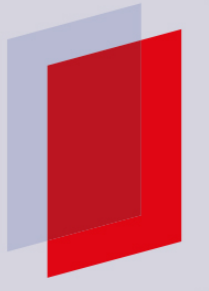

\section{IOP ebooks}

Bringing you innovative digital publishing with leading voices to create your essential collection of books in STEM research. Start exploring the collection - download the first chapter of every title for free. 


\title{
Letter
}

\section{Elastic collision and breather formation of spatiotemporal vortex light bullets in a cubic-quintic nonlinear medium}

\author{
S K Adhikari \\ Instituto de Física Teórica, UNESP_Universidade Estadual Paulista, 01.140-070 São Paulo, São Paulo, \\ Brazil \\ E-mail: adhikari44@yahoo.com
}

Received 6 February 2017, revised 31 March 2017

Accepted for publication 5 April 2017

Published 4 May 2017

\begin{abstract}
The statics and dynamics of a stable, mobile three-dimensional (3D) spatiotemporal vortex light bullet in a cubic-quintic nonlinear medium with a focusing cubic nonlinearity above a critical value and any defocusing quintic nonlinearity are considered. The present study is based on an analytic variational approximation and a full numerical solution of the 3D nonlinear Schrödinger equation. The 3D vortex bullet can propagate with constant velocity. Stability of the vortex bullet is established numerically and variationally. Collision between two vortex bullets moving along the angular momentum axis is considered. At large velocities the collision is quasi-elastic, with the bullets emerging after collision with practically no distortion. At small velocities two bullets coalesce to form a single entity called a breather.
\end{abstract}

Keywords: NLS equation, soliton, vortex bullet

(Some figures may appear in colour only in the online journal)

\section{Introduction}

A one-dimensional (1D) bright soliton with cubic nonlinearity, capable of moving at a constant velocity $[1,2]$, has been observed in nonlinear optics $[1,2]$ in both temporal [3] and spatial [4] varieties. Although a three-dimensional (3D) spatiotemporal soliton cannot be formed with a cubic nonlinearity due to collapse $[1,5]$, the soliton can be stabilized in higher dimensions with a saturable $[5,6]$ or a modified nonlinearity [7], with a cubic-quintic nonlinearity [8], or with a modified dispersion. A two-dimensional (2D) spatiotemporal optical soliton has been observed [9] in a saturable nonlinearity generated by the cascading of quadratic nonlinear processes. A 2D spatial soliton in a cubic-quintic medium has been suggested [10] and realized experimentally [11]. The generation of a stable 2D vortex soliton in a cubic-quintic medium has been suggested [12]. There has also been a study of the dynamics of the vortex pulsed beam in a medium with nonlinearities of opposite sign [13] and of interacting vortices in Bose-Einstein condensates (BEC) [14].

A 3D spatiotemporal optical soliton, commonly known as a light bullet, was realized experimentally in arrays of wave guides [15]. There are many theoretical-numerical and analytical-studies on light bullets using the 3D nonlinear Schrödinger (NLS) equation [1] with a modified nonlinearity [6, 7], nonlinear dissipation [16], and/or dispersion [17]. Dispersion and nonlinearity management can stabilize light bullets in a medium with cubic nonlinearity [18]. Solitons have also been studied in the coupled NLS equation [19]. Recently, we studied [8] the formation of a 3D spatiotemporal light bullet $[5,6]$ in a cubic-quintic medium for a defocusing quintic nonlinearity and a focusing cubic nonlinearity. A cubic-quintic medium is also of experimental interest. A study featuring a polydiacetylene paratoluene sulfonate crystal in the wavelength region near $1600 \mathrm{~nm}$ shows that the refractive index versus input intensity correlation leads to a cubic-quintic form 
of nonlinearity in the NLS equation $[1,20]$. Such a cubicquintic nonlinearity also arises in a low intensity expansion of the saturable nonlinearity used in the pioneering study of light bullets [6].

In this Letter we demonstrate the stabilization of a 3D spatiotemporal vortex (rotating) light bullet in a cubic-quintic medium and study its statics and dynamics, employing variational and numerical solutions of the 3D nonlinear Schrödinger equation. The vortex light bullet is capable of moving without deformation with constant velocity. We study the collision between two vortex light bullets moving along the spinning axis. Such a collision in 3D is expected to be inelastic, with loss of energy. In the present numerical simulation of collision between two vortex light bullets in different parameter domains of nonlinearities and velocities three distinct scenarios are found to take place. At sufficiently large velocities, the collision is found to be quasi-elastic: the two bullets emerge after collision with practically no deformation. At small velocities, the collision is inelastic: the bullets form a single bound entity in an excited state, last for ever and execute oscillation. We call this a breather. In a small domain of intermediate velocities, the bullets coalesce to form a single entity that expands indefinitely, leading to the destruction of the bullets.

We present the 3D NLS equation used in this study in section 2 . In section 3 we present the numerical results for stationary profiles of 3D spatiotemporal vortex light bullets, and numerical tests of stability of the vortex light bullet under a small perturbation. The quasi-elastic nature of collision of two vortex bullets at large velocities and formation of a breather at low velocities are demonstrated by realistic simulation. We end with a summary of our findings in section 4 .

\section{Nonlinear Schrödinger equation: variational formulation}

The 3D NLS equation we describe below to study vortex solitons has application in two areas: in nonlinear optics, where the soliton is known as a spatiotemporal optical vortex bullet, and in BEC. In nonlinear fiber optics the 3D NLS equation is $[1,21]$

$$
\begin{aligned}
& {\left[\mathrm{i} \frac{\partial}{\partial z}+\frac{1}{2 \beta_{0}}\left(\frac{\partial^{2}}{\partial x^{2}}+\frac{\partial^{2}}{\partial y^{2}}\right)+\frac{\beta_{2}}{2} \frac{\partial^{2}}{\partial t^{2}}\right.} \\
& \left.+\gamma|A|^{2}-\kappa|A|^{4}\right] A(x, y, t)=0,
\end{aligned}
$$

where the unit of the parameter $\gamma$ is $\mathrm{W}^{-1} \mathrm{~m}$, that of $\kappa$ is $\mathrm{W}^{-2} \mathrm{~m}^{3}$, that of the intensity $|A|^{2}$ is $\mathrm{W} \mathrm{m}^{-2}$, that of the dispersion parameter $\beta_{2}$ is $\mathrm{ps}^{2} \mathrm{~m}^{-1}$, and that of the propagation constant $\beta_{0}$ is $\mathrm{m}^{-1}$. We define the diffraction length $L_{\mathrm{DF}} \equiv \beta_{0} \omega^{2}$ and dispersion length $L_{\mathrm{DS}} \equiv \tau^{2} / \mid \beta_{2}$, where $\omega$ is the width of the pulse, and $\tau$ is the time scale of the soliton [22]. Now one defines the following dimensionless variables [21]

$$
\begin{aligned}
x & =\frac{x}{\omega}, y=\frac{y}{\omega}, t=\frac{t}{\omega \sqrt{\beta_{0} \beta_{2}}}, z=\frac{z}{L_{\mathrm{DF}}}, \\
\Phi & =\frac{A \sqrt{\gamma L_{\mathrm{DF}}}}{\sqrt{P}_{0}}, p=P_{0}, q=\frac{\kappa P_{0}^{2}}{\gamma^{2} L_{\mathrm{DF}}} .
\end{aligned}
$$

The scale $P_{0}$ is chosen to yield unit norm: $\int|\Phi|^{2} \mathrm{~d} x \mathrm{~d} y \mathrm{~d} t=1$. Using dimensionless variables, one obtains the following NLS equation with self-focusing cubic and self-defocusing quintic nonlinearity [1]

$$
\left[\mathrm{i} \frac{\partial}{\partial z}+\frac{1}{2}\left(\frac{\partial^{2}}{\partial x^{2}}+\frac{\partial^{2}}{\partial y^{2}}+\frac{\partial^{2}}{\partial t^{2}}\right)+p|\Phi|^{2}-q|\Phi|^{4}\right] \Phi(\mathbf{r}, z)=0,
$$

where $\mathbf{r} \equiv\{x, y, t\}, p$ and $q$ are the coefficients of cubic and quintic nonlinearities respectively. In (3) $x, y$ denote transverse extensions, $z$ the propagation distance, and $t$ the time. The quintic nonlinearity of strength $q$ with a negative sign denotes self-defocusing. The plus sign before $|\Phi|^{2}$ denotes a self-focusing cubic nonlinearity.

For a vortex of charge $L$ with circular symmetry in the $x-y$ plane, we can write $\Phi(\mathbf{r}, z)=\phi_{L}(\rho, t, z) \exp (\mathrm{i} L \theta)$, $\rho=\sqrt{x^{2}+y^{2}}, x=\rho \sin \theta, y=\rho \cos \theta$, where the function $\phi_{L}(\rho, t, z)$ is real with the property $\lim _{\rho \rightarrow 0} \phi_{L}(\rho, t, z) \rightarrow \rho^{L}$. This generates an optical pulse with a dark spot at the center $(\rho=0)$ and is called an optical vortex [23]. The wave function $\Phi(\mathbf{r}, z)$ is periodic in $\theta$ with period $2 \pi$ (rotational symmetry). Consequently, recalling

$$
\frac{\partial^{2}}{\partial x^{2}}+\frac{\partial^{2}}{\partial y^{2}}=\frac{\partial^{2}}{\partial \rho^{2}}+\frac{1}{\rho} \frac{\partial}{\partial \rho}+\frac{1}{\rho^{2}} \frac{\partial^{2}}{\partial \theta^{2}},
$$

for unit charge $L=1$, (3) becomes [23]

$$
\begin{gathered}
{\left[\mathrm{i} \frac{\partial}{\partial z}+\frac{1}{2}\left(\frac{\partial^{2}}{\partial \rho^{2}}+\frac{1}{\rho} \frac{\partial}{\partial \rho}+\frac{\partial^{2}}{\partial t^{2}}\right)-\frac{1}{2 \rho^{2}}\right.} \\
\left.+p|\phi|^{2}-q|\phi|^{4}\right] \phi(\rho, t, z)=0,
\end{gathered}
$$

where we have dropped the $L=1$ index from the wave function.

To estimate the order of magnitude of different variables, we consider an infrared beam of wavelength $\lambda=1 \mu \mathrm{m}$ in a nonlinear medium of $\beta_{2}=10^{-2} \mathrm{ps}^{2} \mathrm{~m}^{-1}$, with the time scale $\tau=60 \mathrm{fs}$. Then the beam width $\omega \approx 239 \mu \mathrm{m}$ and the dispersion length $L_{\mathrm{DS}}=36 \mathrm{~cm}$. These numbers are quite similar to those in an experiment on spatiotemporal optical bullets in a planar glass wave-guide [24]. Here we present the results in dimensionless units, which can be converted to actual experimental units using the transformations (2).

The analytic model (5) is also applicable to the case of a vortex soliton in BEC [25]. In that case the mean-field GrossPitaevskii equation describing the $\mathrm{BEC}$ in the presence of attractive two-body and repulsive three-body interactions is given by [26]

$$
\begin{aligned}
\mathrm{i} \hbar \frac{\partial \psi(\mathbf{r}, t)}{\partial t}= & {\left[-\frac{\hbar^{2}}{2 m} \nabla_{\mathbf{r}}^{2}-\frac{4 \pi|a| \hbar^{2} N}{m}|\psi(\mathbf{r}, t)|^{2}\right.} \\
& \left.+\frac{\hbar N^{2} K_{3}}{2}|\psi(\mathbf{r}, t)|^{4}\right] \psi(\mathbf{r}, t),
\end{aligned}
$$

where $m$ is the mass of each atom of the BEC, $\psi(\mathbf{r}, t)$ is the condensate wave function at space point $\mathbf{r}=\{x, y, z\}$ and time $t, \rho=\sqrt{x^{2}+y^{2}}, a$ is the $s$-wave scattering length of atoms, $K_{3}$ is the three-body interaction term, and $N$ is the number of 
atoms. Equation (6) can be written in the following dimensionless form after a redefinition of the variables

$\mathrm{i} \frac{\partial \psi(\mathbf{r}, t)}{\partial t}=\left[-\frac{\nabla_{\mathbf{r}}^{2}}{2}-p|\psi(\mathbf{r}, t)|^{2}+q|\psi(\mathbf{r}, t)|^{4}\right] \psi(\mathbf{r}, t)$,

where $p=4 \pi N, q=m N^{2} K_{3} /\left(2 \hbar a^{4}\right)$, length is scaled in units of $|a|$, time in $m a^{2} / \hbar,|\psi|^{2}$ in units of $|a|^{-3}$. Equations (3) and (7) are mathematically the same, but the interpretation of the various terms in them is distinct. A BEC vortex soliton can be introduced in (7) in a similar fashion as in the case of an optical pulse, viz. (5). In the following we will discuss mostly the case of a spatiotemporal vortex light bullet in cubic-quintic medium. Nevertheless, the similarity of the mathematical models (3) and (7) ensures the the possibility of generating a $3 \mathrm{D}$ vortex soliton in a BEC with repulsive three-body and attractive two-body interactions.

For an analytic understanding of the formation of a spinning light bullet (a vortex soliton of unit charge), we consider the Lagrange variational formulation of an optical pulse [27]. In this axially symmetric problem, a convenient analytic variational approximation of the vortex bullet is $[27,28]$

$$
\begin{aligned}
\phi(\rho, t, z)= & \frac{\pi^{-3 / 4} \rho}{\sigma_{1}^{2}(z) \sqrt{\sigma_{2}(z)}} \exp \left[-\frac{\rho^{2}}{2 \sigma_{1}^{2}(z)}-\frac{t^{2}}{2 \sigma_{2}^{2}(z)}\right. \\
& \left.+\mathrm{i} \alpha(z) \rho^{2}+\mathrm{i} \beta(z) t^{2}\right],
\end{aligned}
$$

where $r^{2}=\rho^{2}+t^{2}, \sigma_{1}(z)$ and $\sigma_{2}(z)$ are radial and axial widths, respectively, and $\alpha(z), \beta(z)$ are corresponding chirps. The (generalized) Lagrangian density corresponding to (5) is given by

$$
\begin{aligned}
\mathcal{L}(\rho, t, z)= & \frac{\mathrm{i}}{2}\left(\phi \dot{\phi}^{*}-\phi^{*} \dot{\phi}\right)+\frac{|\nabla \phi(\rho, t, z)|^{2}}{2}+\frac{|\phi(\rho, t, z)|^{2}}{2 \rho^{2}} \\
& -\frac{p}{2}|\phi(\rho, t, z)|^{4}+\frac{q}{3}|\phi(\rho, t, z)|^{6}
\end{aligned}
$$

where the overhead dot denotes $z$-derivative. Equation (5) can be obtained by extremizing the functional (9) [27]. Consequently, the effective Lagrangian function $\bar{L}\left(\sigma_{1}, \sigma_{2}, \alpha, \beta\right) \equiv 2 \pi \int \mathcal{L}(\rho, t, z) \mathrm{d} t \rho \mathrm{d} \rho$ becomes

$$
\begin{aligned}
\bar{L}\left(\sigma_{1}, \sigma_{2}, \alpha, \beta\right)= & \sigma_{2}^{2}\left(\frac{\dot{\beta}}{2}+\beta^{2}\right)+2 \sigma_{1}^{2}\left(\dot{\alpha}+2 \alpha^{2}\right)+\frac{1}{\sigma_{1}^{2}} \\
& +\frac{1}{4 \sigma_{2}^{2}}-\frac{p \pi^{-3 / 2}}{8 \sqrt{2} \sigma_{1}^{2} \sigma_{2}}+\frac{2 q \pi^{-3}}{81 \sqrt{3} \sigma_{1}^{4} \sigma_{2}^{2}} .
\end{aligned}
$$

The variational parameters $\nu \equiv \sigma_{1}, \sigma_{2}, \alpha, \beta$ are obtained from the Euler-Lagrangian equations

$$
\frac{\mathrm{d}}{\mathrm{d} z} \frac{\partial \bar{L}}{\partial \dot{\nu}}=\frac{\partial \bar{L}}{\partial \nu}
$$

After some straightforward algebra the four Euler-Lagrangian equations lead to the following dynamical equations for the widths:

$$
\begin{aligned}
& \frac{1}{\sigma_{1}^{3}}-\frac{p \pi^{-3 / 2}}{8 \sqrt{2} \sigma_{1}^{3} \sigma_{2}}+\frac{4 q \pi^{-3}}{81 \sqrt{3} \sigma_{1}^{5} \sigma_{2}^{2}}=\ddot{\sigma}_{1}, \\
& \frac{1}{\sigma_{2}^{3}}-\frac{p \pi^{-3 / 2}}{4 \sqrt{2} \sigma_{1}^{2} \sigma_{2}^{2}}+\frac{8 q \pi^{-3}}{81 \sqrt{3} \sigma_{1}^{4} \sigma_{2}^{3}}=\ddot{\sigma}_{2} .
\end{aligned}
$$

The stationary profile of the vortex bullet is obtained by setting the $z$-derivatives on the right-hand sides of (12) and (13) [27]:

$$
\begin{aligned}
& \frac{1}{\sigma_{1}^{3}}-\frac{p \pi^{-3 / 2}}{8 \sqrt{2} \sigma_{1}^{3} \sigma_{2}}+\frac{4 q \pi^{-3}}{81 \sqrt{3} \sigma_{1}^{5} \sigma_{2}^{2}}=0, \\
& \frac{1}{\sigma_{2}^{3}}-\frac{p \pi^{-3 / 2}}{4 \sqrt{2} \sigma_{1}^{2} \sigma_{2}^{2}}+\frac{8 q \pi^{-3}}{81 \sqrt{3} \sigma_{1}^{4} \sigma_{2}^{3}}=0 .
\end{aligned}
$$

Equations (14) and (15) correspond to the global minimum of a conserved $\alpha$ - and $\beta$-independent effective Lagrangian $L\left(\sigma_{1}, \sigma_{2}\right) \equiv \bar{L}\left(\sigma_{1}, \sigma_{2}, \alpha=0, \beta=0\right): \quad \partial L / \partial \sigma_{1}=\partial L / \partial \sigma_{2}=0$. The function $L\left(\sigma_{1}, \sigma_{2}\right)$ describes the Lagrangian dynamics of the widths $\sigma_{1}, \sigma_{2}$ and is independent of the generalized velocities $\dot{\sigma}_{1}, \dot{\sigma}_{2}$

\section{Numerical results}

The 3D NLS equation (5) is generally solved by the splitstep Crank-Nicolson [29] and Fourier spectral [30] methods. The split-step Crank-Nicolson method in Cartesian coordinates is employed in the present study. We use $\mathbf{r}=\{x, y, t\}$ step of 0.05-0.016, a $z$ step of 0.0005-0.0000025 [29] and the number of $\mathbf{r}$ discretization points $128-320$. There are different $\mathrm{C}$ and FORTRAN programs for solving the NLS-type equations [29, 31] and one should use the appropriate one. We use both imaginary- and real-z propagation [29] for numerical solution of the 3D NLS equation. The imaginary- $z$ propagation is appropriate to find the stationary state and the real- $z$ propagation for the dynamics. In the imaginary- $z$ propagation the initial state was taken as in (8).

A stable bullet corresponds to a global minimum of the conserved effective Lagrangian $L\left(\sigma_{1}, \sigma_{2}\right)$ at a negative value. To demonstrate the appearance of a global minimum, we show in figure 1 the two-dimensional contour plot of the Lagrangian $L\left(\sigma_{1}, \sigma_{2}\right)$ in the $\sigma_{1}-\sigma_{2}$ plane for (a) $p=q=200$, (b) $p=200$, $q=400$, (c) $p=100, q=200$, and (d) $p=q=100$, where we illustrate the region with negative Lagrangian; the Lagrangian is positive outside this region. The Lagrangian $L\left(\sigma_{1}, \sigma_{2}\right)$ goes to zero as $\sigma_{1}, \sigma_{2} \rightarrow \infty$. At the origin, $\sigma_{1}, \sigma_{2} \rightarrow 0$, and the Lagrangian $L\left(\sigma_{1}, \sigma_{2}\right) \rightarrow \infty$, which guarantees the absence of a collapsed state at the origin. The repulsive quintic nonlinearity contributes positively to the Lagrangian; so do the first two terms on the right-hand side of (10). To make the Lagrangian (10) negative, the cubic nonlinearity coefficient $p$ has to be larger than a critical value, e.g. $p>p_{\text {crit }}$, when the minimum of the Lagrangian could be negative, corresponding to a stable vortex bullet. For $p<p_{\text {crit }}$ the optical pulse is too repulsive to form a vortex bullet. In figure 2(a) we show the variational 
(a)

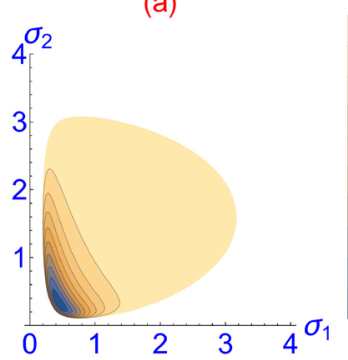

(c)
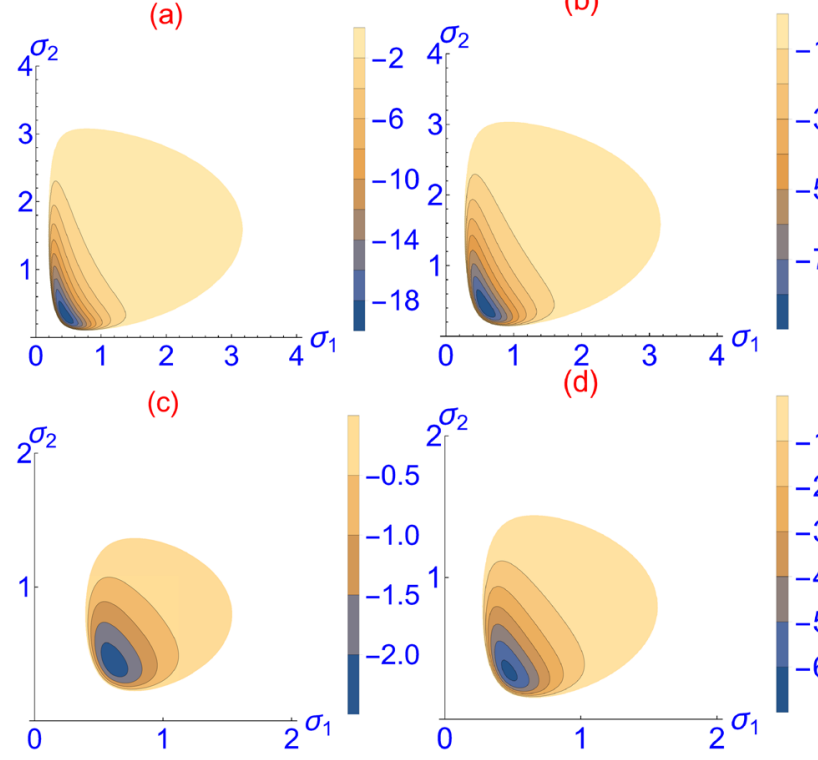

(d)

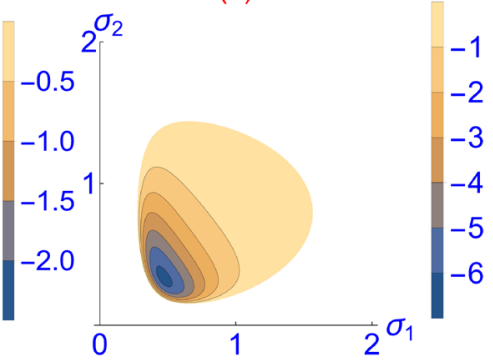

Figure 1. Contour plot of Lagrangian $L\left(\sigma_{1}, \sigma_{2}\right)(10)$ as a function of $\sigma_{1}$ and $\sigma_{2}$ for (a) $p=200, q=200$, (b) $p=200, q=400$, (c) $p=100, q=200$, (d) $p=100, q=100$. The Lagrangian is negative in the shaded region and positive outside.

values for $p_{\text {crit }}$ versus $q$. We compare the numerical and variational results for the root-mean-square (rms) sizes $x_{\text {rms }}$ and $t_{\mathrm{rms}}$ in figure 2(b) and Lagrangian $|L|$ in figure 2(c), for $q=100$ and 200. The variational Lagrangian is calculated using (9) with the numerically obtained $\sigma_{1}, \sigma_{2}$, corresponding to the minimum of Lagrangian (10) given by (12) and (13). The variational $x_{\mathrm{rms}} \equiv \sigma_{1}$. The rms sizes $x_{\mathrm{rms}}, t_{\mathrm{rms}}$ and Lagrangian $L$ of figures 2(b)-(c), respectively, increase with increasing $q$ value, corresponding to repulsion, and decrease with increasing $p$ value, corresponding to attraction.

To study the density distribution of the spatiotemporal vortex light bullets we define the reduced 1D and 2D densities by

$$
\begin{aligned}
& \delta_{1 \mathrm{D}}(x)=\int \mathrm{d} t \mathrm{~d} y|\phi(\mathbf{r})|^{2}, \\
& \delta_{2 \mathrm{D}}(x, y)=\int \mathrm{d} t|\phi(\mathbf{r})|^{2} .
\end{aligned}
$$

In figure 3 we show these reduced densities $\delta_{1 \mathrm{D}}(x)$ and $\delta_{2 \mathrm{D}}(x, 0)$ as obtained from numerical and variational calculations for various cubic nonlinearity coefficient $p$ and quintic nonlinearity coefficient $q$. The corresponding Lagrangian values are also exhibited. For a fixed defocusing quintic nonlinearity coefficient $q(=200)$, the vortex bullet is more compact with the increase of focusing nonlinearity coefficient $p$, resulting in more attraction-as can be found in figures 3(a) and (c). For fixed focusing nonlinearity coefficient $p=200$ and 100, the light bullet is more compact, with the decrease of defocusing nonlinearity coefficient $q$ resulting in less repulsion-as found in figures 3(a)-(b) and in $3(\mathrm{c})-(\mathrm{d})$ respectively.

The negative-Lagrangian finite well of figure 1 trapping the spatiotemporal vortex light bullet guarantees its stability because of Lagrangian conservation. Now we present a numerical test of stability of a vortex bullet. For this purpose we consider the vortex bullet shown in figure 3(c) with $p=100$ and $q=200$ as calculated by imaginary- $z$ propagation. Using the imaginary- $z$ profile as the initial state, we perform numerical simulation by real- $z$ propagation. The real- $z$ propagation shows steady breathing oscillation of the vortex bullet for a large $z$ propagation. In figures $4(\mathrm{a})-(\mathrm{c})$ we show the 3D isodensity contour of the vortex bullet at $z=0,6$ and 12. The vortex core remains intact in this propagation. No transverse instability [1] of the vortex core was found. In figure 4(d) we show the steady (monopole breathing) oscillation in the rms $x$ and $t$ sizes $x_{\text {rms }}$ and $t_{\text {rms }}$ versus propagation distance $z$ during real- $z$ propagation. The steady continued oscillation of the vortex bullet over a long distance of propagation establishes the stability of the bullet. The real- $z$ simulation was performed in full 3D space, without assuming spherical symmetry, to guarantee the stability in full 3D Cartesian space.

A collision between two analytic 1D solitons is truly elastic [1] due to the conservation laws (of energy, momentum), and such solitons pass through each other without deformation at any incident velocity. A collision between two 3D spatiotemporal vortex bullets is expected to be inelastic in general, due to loss of kinetic energy leading to their deformation. However, under ideal conditions at large velocities the collision between two spatiotemporal vortex bullets can be quasi-elastic. Under these conditions, the kinetic energy of the colliding vortex bullets is much larger than the internal interaction energies, and the duration of the encounter in $z$ is small. At the other extreme, when the kinetic energies of the colliding vortex bullets are much smaller than the internal binding energies, the encounter is controlled solely by the internal interactions, and the two vortex bullets form a bound entity after collision, called a breather.

To test the nature of collision between the present spatiotemporal vortex light bullets, we study the frontal head-on collision of the same along the angular momentum axis $(t)$. A moving bullet with velocity $v$ along the $t$ axis can be generated by multiplying the bullet wave function by $\exp (i v t)$ and performing real- $z$ simulation with this function. The imaginary- $z$ profile of the light bullet shown in figure 3(c) with $p=100$, $q=200$ is used as the initial function in the real $-z$ simulation of collision, with two identical bullets placed at $x= \pm 1.45$ initially at $z=0$. The vortex bullets are set in motion along the $t$ axis, in opposite directions, with velocity $v=43$. To illustrate the dynamics upon real- $z$ simulation, we plot the $3 \mathrm{D}$ density contour $|\phi(\mathbf{r}, z)|^{2}$ at different values of propagation distance $z$ in figure 5 . In this case, the kinetic energy $v^{2} / 2 \approx 924$ is much larger than the Lagrangian $(|L| \approx 2.5)$; the collision is found to be quasi-elastic, and when considering the $3 \mathrm{D}$ nature of the collision the distortion of the vortex bullets after collision is found to be insignificant.

To study the inelastic collision at very small velocities we consider two compact bullets with $p=200, q=200$, place them at $t= \pm 2$ and set them in motion with velocity $v=0.1$, in opposite directions, along the $t$ axis. The dynamics is illustrated by a plot of the time evolution of $1 \mathrm{D}$ density $\delta_{1 \mathrm{D}}(t, z) \equiv \int \mathrm{d} x \mathrm{~d} y|\phi(\mathbf{r}, z)|^{2}$ versus $t, z$ in figure 6 (a) and the corresponding contour plot 

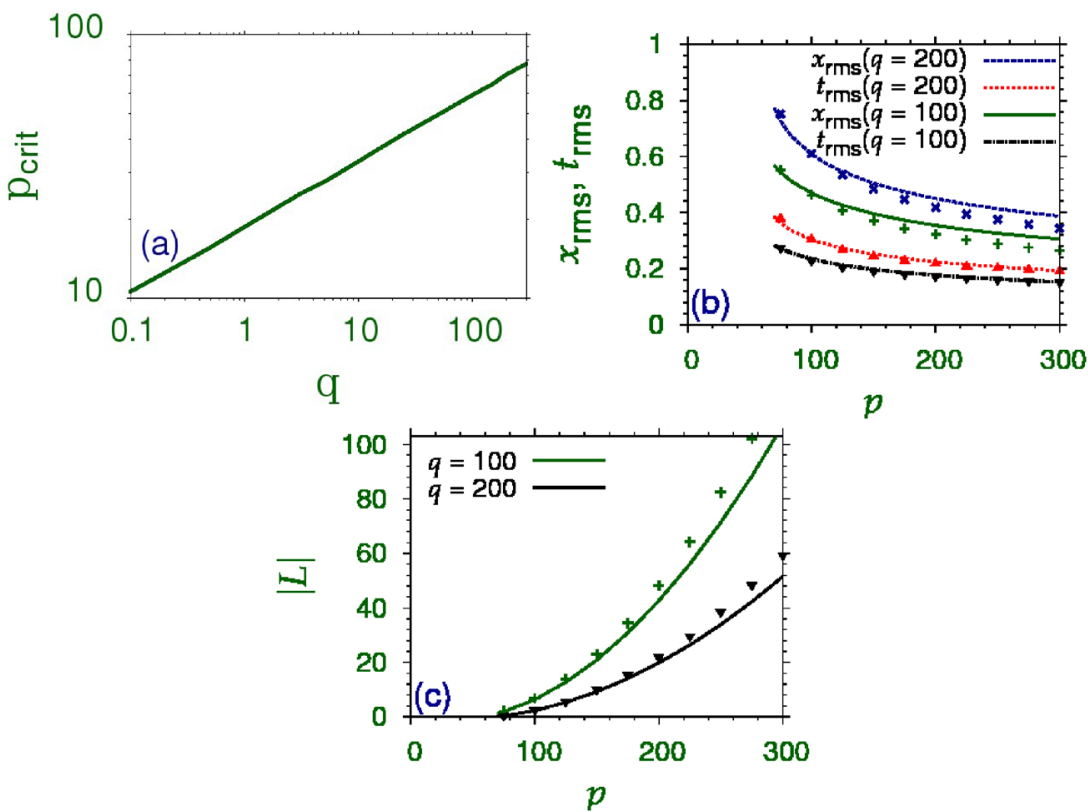

Figure 2. (a) Variational results for $p_{\text {crit }}$ versus $q$. A vortex bullet with negative Lagrangian can be formed for $p>p_{\text {crit }}$ (b) Variational (line) and numerical (points) results for rms sizes $x_{\text {rms }}$ and $t_{\text {rms }}$ versus cubic nonlinearity coefficient $p$ for quintic nonlinearity coefficient $q=100$ and 200. (c) Variational (line) and numerical (points) results for Lagrangian $L$ versus cubic nonlinearity coefficient $p$ for quintic nonlinearity coefficient $q=100$ and 200 .
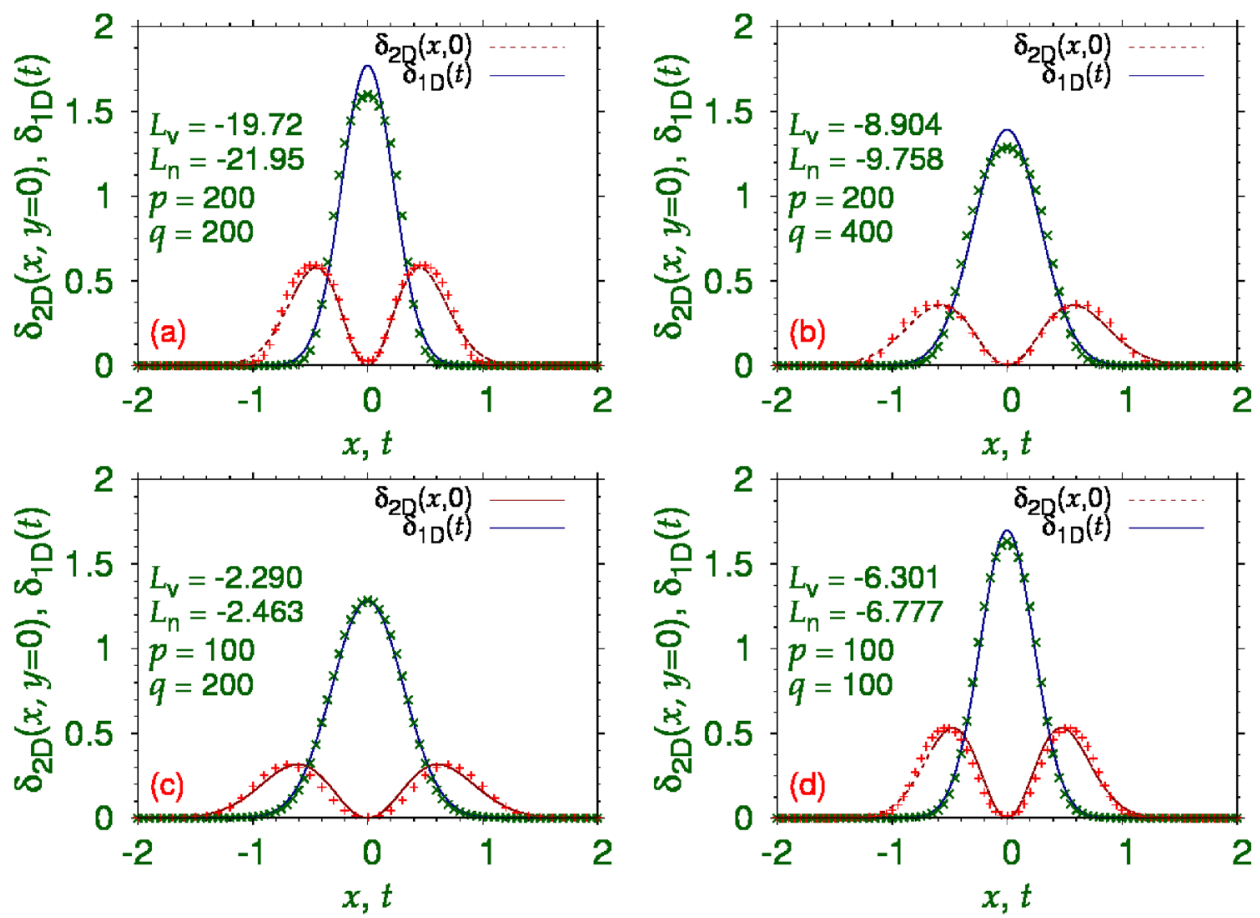

Figure 3. Numerical (line) and variational (points) reduced densities $\delta_{1 \mathrm{D}}(x)$ and $\delta_{2 \mathrm{D}}(x, y=0)$ for different cubic nonlinearity coefficient $p$ and quintic nonlinearity coefficient $q$ : (a) $p=q=200$, (b) $p=200, q=400$, (c) $p=100, q=200$ and (d) $p=q=100$

is shown in figure 6(b). The two vortex bullets come close to each other at $t=0$ coalesce to form a breather and never separate again. The combined bound system remains at rest at $t=0$, continuing in a small breathing oscillation because of a small amount of liberated kinetic energy, which creates the breather in an excited state. The observation of an oscillating breather has been reported some time ago in dissipative systems [32]. In this case the kinetic energy $v^{2} / 2 \approx .005$ is insignificant compared to the Lagrangian $(|L| \approx 21)$, and the collision is fully inelastic with destruction of individual bullets.

However, as the velocity $v$ is reduced from $v \approx 40$ (elastic collision scenario presented in figure 5) to $v \approx 0.1$ (breather 

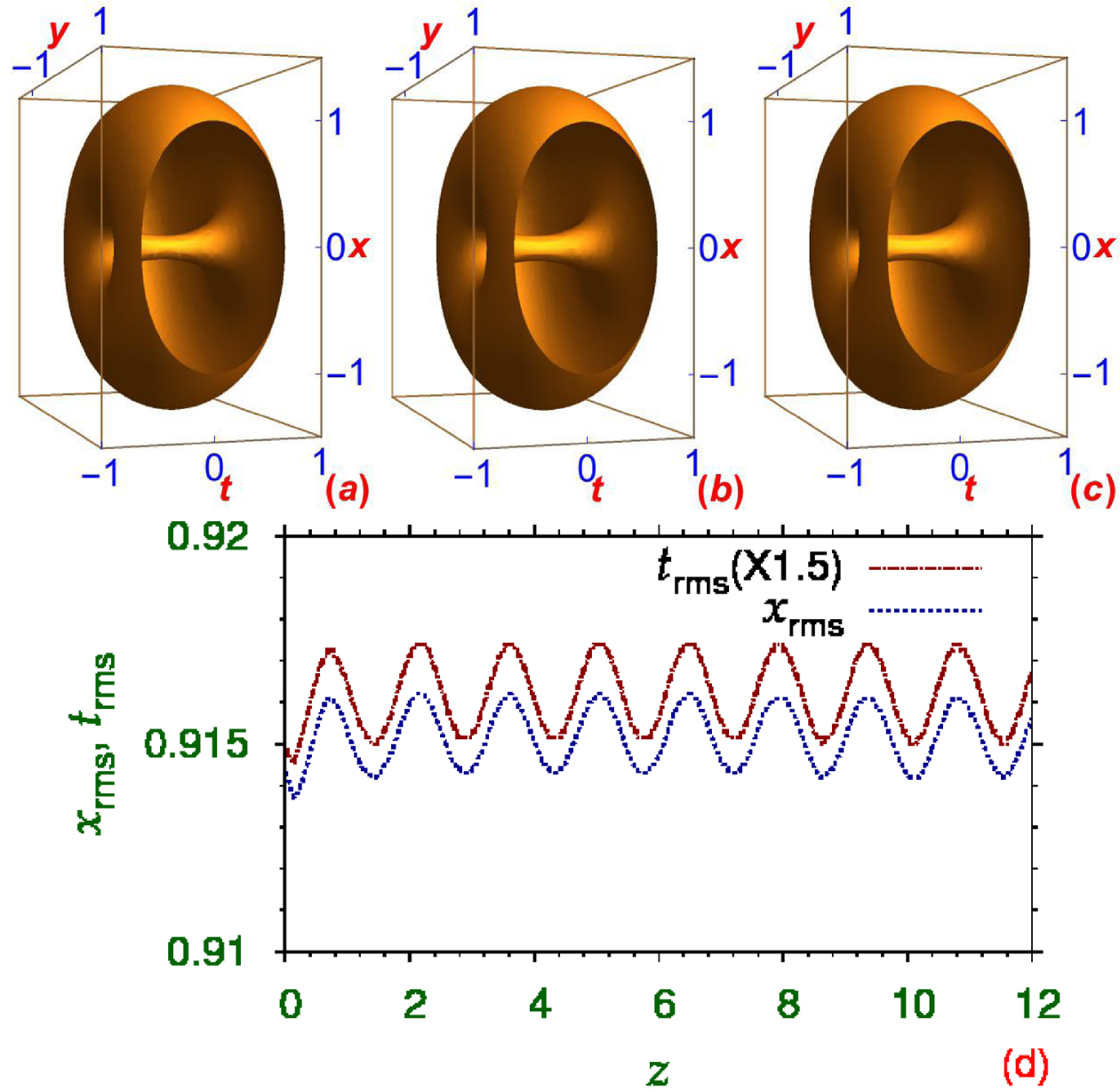

Figure 4. Real-z evolution of the vortex bullet with $p=100$ and $q=200$ by isodensity contour plot of $|\phi(\mathbf{r}, z)|^{2}$ at (a) $z=0$, (b) 6 , (c) 12 . The dimensionless density on the contour is 0.02 . (d) The rms sizes $x_{\mathrm{rms}}$ and $t_{\mathrm{rms}}$ versus $z$ during real- $z$ evolution.
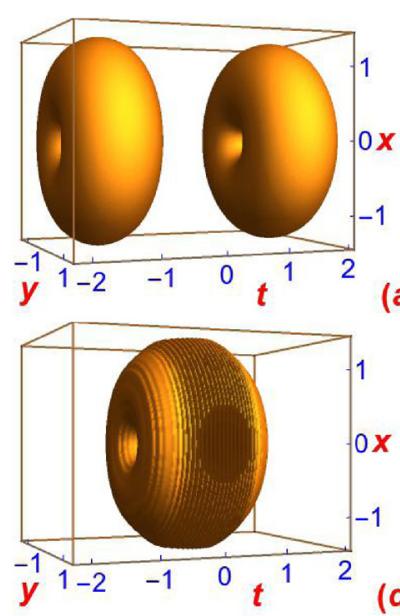

(a)
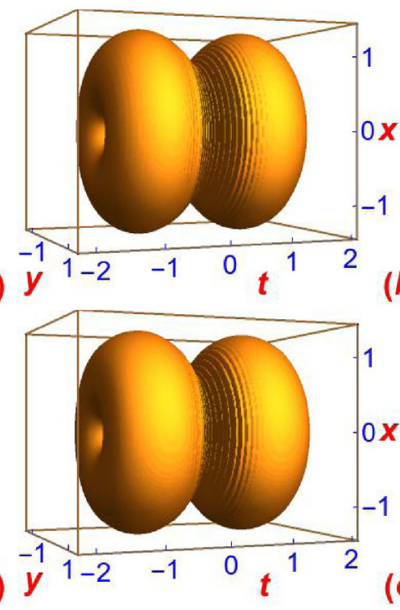

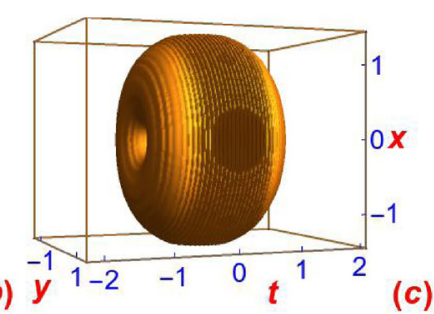

(c)

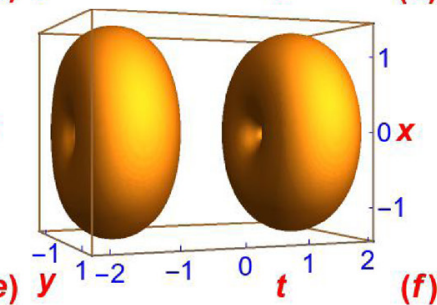

Figure 5. Collision dynamics of two vortex bullets, each with $p=100, q=200$, placed at $t= \pm 1.45$ at $z=0$ and set into motion in opposite directions along the $t$ axis with the velocity of $v=43$, illustrated by isodensity contours at (a) $z=0,(\mathrm{~b})=0.0135$, (c) $=0.027$, (d) $=0.0405$, (e) $=0.054$, (f) $=0.0675$. The density on the contour is 0.02 .

formation as in figure 6), a distortion of the vortex bullets takes place after collision, with eventual destruction of the vortex bullets. This is illustrated in figure 7, where apart from the two trajectories of the vortex bullets after collision a central peak can be seen at $t=0$. On further reduction of the initial velocity, the central peak at $t=0$ becomes more pronounced, and the outer tracks less prominent. Eventually, at very small velocities only the central peak corresponding to the formation of a breather after collision prevails, viz. figure 6 . 

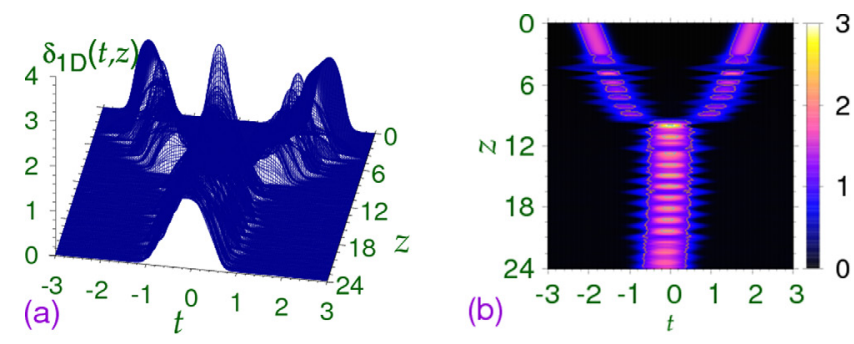

Figure 6. (a) The $1 \mathrm{D}$ density $\delta_{1 \mathrm{D}}(t, z)$ and (b) its $2 \mathrm{D}$ contour plot during the collision of two vortex bullets with $p=q=200$ placed at $t= \pm 2$ at $z=0$ and set into motion in opposite directions along the $t$ axis with the velocity $v=0.1$, and the formation of a breather upon real- $z$, simulation.
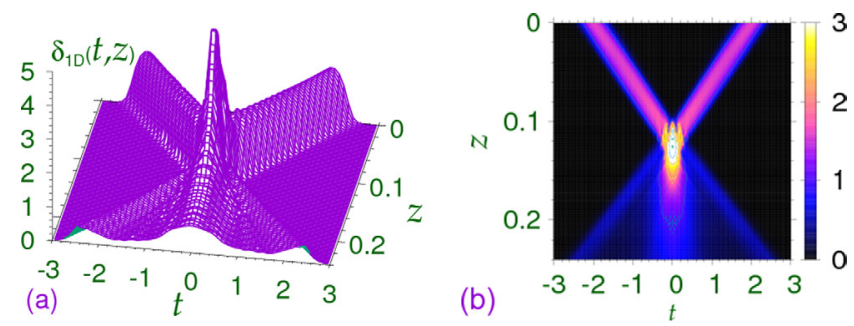

Figure 7. (a) The $1 \mathrm{D}$ density $\delta_{1 \mathrm{D}}(t, z)$ and (b) its $2 \mathrm{D}$ contour plot during the collision of two vortex bullets with $p=q=200$ placed at $t= \pm 2$ at $z=0$ and set into motion in opposite directions along the $t$ axis with the velocity $v=20$, and the destruction of the bullets upon real-z simulation. After collision the two bullets are destroyed and an expanding pulse results upon $z$ evolution.

\section{Summary}

To summarize, we demonstrate the formation of a stable 3D spatiotemporal vortex bullet with cubic-quintic nonlinearity, employing a variational approximation and full 3D numerical solution of the NLS equation. The statical properties of the bullet are studied by a variational approximation and a numerical imaginary- $z$ solution of the 3D NLS equation. The cubic nonlinearity is taken as focusing Kerr type above a critical value, whereas the quintic nonlinearity is defocusing. The dynamical properties are studied by a real- $z$ solution of the NLS equation. In the 3D spatiotemporal case, the vortex light bullet can move with a constant velocity. At large velocities, the collision between the two spatiotemporal vortex light bullets is quasi-elastic, with no visible deformation of the final bullets. At small velocities, the collision is inelastic with the formation of a breather after collision. At medium velocities the bullets can be destroyed after collision.

\section{Acknowledgments}

We thank the Fundação de Amparo à Pesquisa do Estado de São Paulo (Brazil) (Project: 2012/00451-0) and the Conselho Nacional de Desenvolvimento Científico e Tecnológico (Brazil) (Project: 303280/2014-0) for support.

\section{References}

[1] Kivshar Y S and Agrawal G 2003 Optical Solitons: From Fibers to Photonic Crystals (San Diego: Academic)

[2] Kivshar Y S and Malomed B A 1989 Rev. Mod. Phys. 61763 Mihalache D 2014 Rom. J. Phys. 59295

[3] Di Trapani P, Caironi D, Valiulis G, Dubietis A, Danielius R and Piskarskas A 1998 Phys. Rev. Lett. 81570

[4] Kang J U, Stegeman G I and Aitchison J S 1996 Opt. Lett. 21189 Kang J U, Stegeman G I, Aitchison J S and Akhmediev N 1996 Phys. Rev. Lett. 763699

[5] Silberberg Y 1990 Opt. Lett. 151282

[6] Akhmediev N and Soto-Crespo J M 1993 Phys. Rev. Lett. 471358

Skryabin D V and Firth W J 1998 Opt. Commun. 14879 Edmundson D E and Enns R H 1992 Opt. Lett. 17586

Fibich G and Ilan B 2004 Opt. Lett. 29887

Torner L and Kartashov Y V 2009 Opt. Lett. 341129

Kanashov A A and Rubenchik A M 1981 Physica D 4122

Mihalache D, Mazilu D, Crasovan L-C, Towers I, Buryak A V, Malomed B A, Torner L, Torres P J and Lederer F 2002 Phys. Rev. Lett. 88073902

[7] McLeod R, Wagner K and Blair S 1995 Phys. Rev. A 523254 Mihalache D, Mazilu D, Crasovan L-C, Torner L, Malomed B A and Lederer F 2000 Phys. Rev. E 627340

[8] Adhikari S K 2016 Phys. Rev. E 94032217

[9] Liu X, Qian L J and Wise F W 1999 Phys. Rev. Lett. 824631

[10] Quiroga-Teixeiro M L, Berntson A and Michinel H 1999 J. Opt. Soc. Am. B 161697

[11] Falcão-Filho E L, de Araujo C B, Boudebs G, Leblond H and Skarka V 2013 Phys. Rev. Lett. 110013901

[12] Berezhiani V I, Skarka V and Aleksić N B 2001 Phys. Rev. E 64057601

[13] Vlasov R A et al 2005 Quantum Electron. 35947

[14] Adhikari S K 2003 New J. Phys. 5137

[15] Minardi S et al 2010 Phys. Rev. Lett. 105263901

[16] Grelu P, Soto-Crespo J and Akhmediev N 2005 Opt. Express 139352

Aleksić N B, Skarka V, Timotijević D V and Gauthier D 2007 Phys. Rev. A 75061802

Kamagate A, Grelu P, Tchofo-Dinda P, Soto-Crespo J M and Akhmediev N 2009 Phys. Rev. E 79026609

Chen S 2012 Phys. Rev. A 86033829

Mihalache D, Mazilu S, Lederer F, Kartashov Y V, Crasovan L-C, Torner L and Malomed B A 2006 Phys. Rev. Lett. 97073904

Mihalache D, Mazilu D, Lederer F, Leblond H and Malomed B A 2009 Eur. Phys. J. Spec. Top. 173245

[17] Adhikari S K 2005 Phys. Rev. E 71016611

[18] Matuszewski M, Infeld E, Malomed B A and Trippenbach M 2006 Opt. Commun. 25949

[19] Radhakrishnan R, Lakshmanan M and Hietarinta J 1997 Phys. Rev. E 562213

Kanna T and Lakshmanan M 2001 Phys. Rev. Lett. 865043

Radhakrishnan R and Lakshmanan M 1995 J. Phys. A: Math. Gen. 282683

Adhikari S K 2005 Phys. Lett. A 346179

[20] Lawrence B, Torruellas W E, Cha M, Sundheimer M L, Stegeman G I, Meth J, Etemad S and Baker G 1994 Phys. Rev. Lett. 73597

[21] Agrawal G P 2011 J. Opt. Soc. Am. B 28 A1

[22] Malomed B A, Mihalache D, Wise F and Torner L 2005 J. Opt. B 7 R53

[23] Desyatnikov A S, Kivshar Y S and Torner L 2005 Prog. Opt. 47291 
Swartzlander G A Jr and Law C T 1992 Phys. Rev. Lett. 692503

Marrucci L, Manzo C and Paparo D 2006 Phys. Rev. Lett. 96163905

Williams M D, Coles M M, Bradshaw D S and Andrews D L 2014 Phys. Rev. A 89033837

Shen Y, Campbell G T, Hage B, Zou H, Buchler B C and Lam P K 2013 J. Opt. 15044005

Motzek K, Kaiser F, Salgueiro J R, Kivshar Y and Denz C 2004 Opt. Lett. 292285

Law C T, Zhang X and Swartzlander G A 2000 Opt. Lett. 2555

[24] Eisenberg H S, Morandotti R, Silberberg Y, Bar-Ad S, Ross D and Aitchison J S 2001 Phys. Rev. Lett. 87043902

[25] Mihalache D 2017 Rom. Rep. Phys. 69403

Yukalov V I and Yukalova E P 2016 Laser Phys. 26045501

[26] Adhikari S K 2017 Laser Phys. Lett. 14025501

Adhikari S K 2017 Phys. Rev. A 95023606

[27] Anderson D 1983 Phys. Rev. A 273135

Anderson D, Lisak M and Reichel T 1988 Phys. Rev. A 381618

[28] Perez-Garcia V M, Michinel H, Cirac J I, Lewenstein M and Zoller P 1997 Phys. Rev. A 561424
[29] Muruganandam P and Adhikari S K 2009 Comput. Phys. Commun. 1801888

Vudragović D, Vidanović I, Balaž A, Muruganandam P and Adhikari S K 2012 Comput. Phys. Commun. 1832021

Young-S L E, Vudragović D, Muruganandam P, Adhikari S K and Balaž A 2016 Comput. Phys. Commun. 204209

[30] Muruganandam P and Adhikari S K 2003 J. Phys. B: At. Mol. Phys. 362501

[31] Satarić B, Slavnić V, Belić A, Balaž A, Muruganandam P and Adhikari S K 2016 Comput. Phys. Commun. 200411

Loncar V, Balaž A, Bogojević A, Skrbić S, Muruganandam P and Adhikari S K 2016 Comput. Phys. Commun. 200406

Loncar V, Young-S L E, Skrbić S, Muruganandam P, Adhikari S K and Balaž A 2016 Comput. Phys. Commun. 20919 o

Kishor Kumar R, Young-S L E, Vudragović D, Balaž A, Muruganandam P and Adhikari S K 2015 Comput. Phys. Commun. 195117

[32] Soto-Crespo J M, Akhmediev N and Grelu P 2006 Phys. Rev. E 74046612 